\title{
A union of BFF (Bayesian, frequentist and fiducial) inferences by confidence distribution and Monte-Carlo based inference
}

\author{
Dr. Min-ge Xie \\ Rutgers, The State University of New Jersey \\ USA
}

This talk reviews several new developments on confidence distribution and provides a comparative study of the BFF inferences from a unique simulation (artificial data sampling) angle. It is shown that the connections between Bayesian and frequentist inferences (including modern Bayesian computing procedures, bootstrap and many other resampling and simulation methods) are much more congruous and coherent than what have been perceived in the scientific community. Specifically, we will discuss the ideas of uncertainty matching by CD-random variable, by bootstrapping, by fiducial samples and also by Bayesian posterior samples, in which the uncertainty by a model population is matched with the uncertainty from the same or a similar artificial data generation scheme. A general theory of uncertainty matching for exact and asymptotic inference is provided. The development does not rely on the specification of likelihood function and it is especially effective for different inference problems involving discrete parameters where the large sample central limit theorem does not apply. The development is illustrated to solve the well-known post-selection inference problem in high dimensional regression model by jointly creating confidence sets of selected models and model coefficients. 